\section{E-126 ENDOVASCULAR TREATMENT DECISION IN ACUTE STROKE IN PATIENTS WITH SEVERE COMORBIDITIES - INSIGHTS FROM AN INTERNATIONAL MULTIDISCIPLINARY SURVEY}

${ }^{1} \mathrm{~J}$ Ospel ${ }^{*},{ }^{2} \mathrm{~N}$ Kashani, ${ }^{3} \mathrm{~J} \mathrm{Heo},{ }^{4} \mathrm{M}$ Cherian, ${ }^{5} \mathrm{~B}$ Kim, ${ }^{6} \mathrm{~A}$ Rabinstein, ${ }^{7} \mathrm{U}$ Fischer, ${ }^{8} \mathrm{~B}$ Baxter, ${ }^{9} \mathrm{M}$ Foss, ${ }^{10} \mathrm{~F}$ Turjman, ${ }^{11} \mathrm{P}$ Sylaia, ${ }^{12} \mathrm{~S}$ Yoshimura, ${ }^{13} \mathrm{~W}$ Kunz, ${ }^{14} \mathrm{~B}$ Campbell, ${ }^{9} \mathrm{~A}$ Wilson, ${ }^{9} \mathrm{M}$ Almekhlafi, ${ }^{15} \mathrm{G}$ Saposnik, ${ }^{9} \mathrm{M}$ Hill, ${ }^{9} \mathrm{M}$ Goyal, ${ }^{9} \mathrm{~B}$ Menon. ${ }^{1}$ Radiology, University Hospital Basel, Basel, Switzerland; ${ }^{2}$ Radiology, University of Calgary, Basel, AB, Canada; ${ }^{3}$ Neurology, Yonsei University, Seoul, Seoul, Korea, republic of; ${ }^{4}$ Radiology, Kovai Medical center, Tamil Nadu, India; ${ }^{5}$ St. Mary's Hospital Seoul, Seoul, Korea, republic of; ${ }^{6}$ Radiology, Mayo Clinic Rochester, Rochester, MN; 'Radiology, University Hospital Bern (Inselspital), Bern, Switzerland; ${ }^{8}$ Radiology, Erlanger Hospital Chattanooga, Chattanooga, TN; ${ }^{9}$ Radiology, University of Calgary, Calgary, AB, Canada; ${ }^{10}$ Radiology, Centre Hospitalier Universitaire de Lyon, Lyon, Francel; ${ }^{11}$ Radiology, Sree Chitra Tirunal Institute for Medical Sciences and Technology, Thiruvananthapuram, India; ${ }^{12}$ Neurosurgery, Hyogo College of Medicine, Hyogo, Japan; ${ }^{13}$ Radiology, University Hospital Munich, Munich, Germany; ${ }^{14}$ Neurology, Royal Melbourne Hospital, University of Melbourne, Melbourne, Australia; ${ }^{15}$ Radiology, University of Toronto, Toronto, ON, Canada

\subsection{6/neurintsurg-2019-SNIS.201}

Background Endovascular therapy is a highly effective treatment in previously-independent patients. In patients with severe comorbidities, the risks and benefits of endovascular treatment have to be balanced with the life expectancy and treatment risks. We explored the attitude of physicians towards endovascular treatment decision in patients with severe physical comorbidities.

Methods We conducted an international cross-sectional survey among 607 stroke physicians and interventionalists to understand their current practice and therapy decision-making in acute stroke. Participants were asked to give their treatment approach to 10 out of 22 randomly assigned case scenarios, 4 of them involving severe comorbidities (A: heart failure, COPD and renal insufficiency requiring dialysis, B: Stage IV metastatic prostate cancer, C: Non-metastatic prostate cancer with hormonal treatment, D: Rheumatoid arthritis requiring care in a nursing home). In all of those 4 scenarios, the patients had proximal ICA/MCA occlusions and were otherwise EVT-eligible.

Results In total, 6070 responses for 22 scenarios were obtained (276 responses for scenario A, 275 for scenarios B and D, 277 for scenario C). 92\% of respondents decided in favor of EVT in scenario A, 72.7\% in scenario B, $80.5 \%$ in scenario C and $90.2 \%$ in scenario D. Except of scenario B, those proportions were slightly higher than the average overall EVT decision rate (75.2\%).

Conclusion If EVT criteria are otherwise fulfilled, moderate and even severe physical comorbidities were not considered as reasons to refrain from endovascular therapy in this survey.

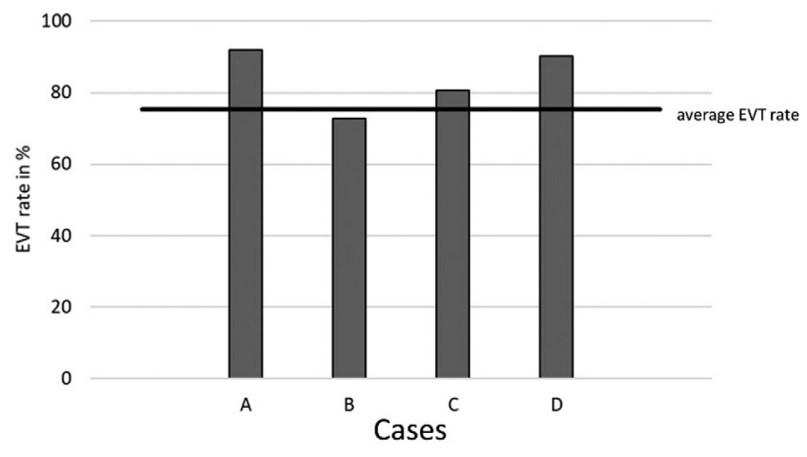

Abstract E-126 Figure 1
Disclosures J. Ospel: None. N. Kashani: None. J. Heo: None. M. Cherian: None. B. Kim: None. A. Rabinstein: None. U. Fischer: None. B. Baxter: None. M. Foss: None. F. Turjman: None. P. Sylaia: None. S. Yoshimura: None. W. Kunz: None. B. Campbell: None. A. Wilson: None. M. Almekhlafi: None. G. Saposnik: None. M. Hill: None. M. Goyal: None. B. Menon: 1; C; unrestricted research grant by Stryker to the University of Calgary.

\section{E-127 MECHANICAL THROMBECTOMY. RETROSPECTIVE REVIEW OF INCIDENCES AND COMPLICATIONS}

${ }^{1} \mathrm{M}$ de Miquel ${ }^{*},{ }^{1} \mathrm{~L}$ Aja, ${ }^{1} \mathrm{~S}$ Aixut, ${ }^{1} \mathrm{R}$ Barranco, ${ }^{1} \mathrm{O}$ Chirife, ${ }^{2} \mathrm{P}$ Cardona, ${ }^{3} \mathrm{H}$ Quesada, ${ }^{1} \mathrm{~B}$ Lara, ${ }^{2} \mathrm{~A}$ Paipa. ${ }^{1}$ Neuroradiology, Hospital Universitari de Bellvitge, L'Hospitalet de Llobregat, Spain; ${ }^{2}$ Neurology, Hospital Universitari de Bellvitge, L'Hospitalet de Llobregat, Spain; ${ }^{3}$ Neuroology, Hospital Universitari de Bellvitge, L'Hospitalet de Llobregat, Spain

10.1136/neurintsurg-2019-SNIS.202

Introduction and purpose Mechanical thrombectomy is accepted as the best treatment when possible in cases of ischemic stroke. Since indications are getting broader in terms of age, time of stroke evolution or NIHSS and ASPECTS records, we frequently face the decision of the convenience to treat borderline patients, for example oligosymptomatic cases. We aimed to review our complications in terms of frequency, age and location in order to help us in taking treatment decisions.

Material and methods This study is based in a retrospective review of our database. Since 2010 we have registered 629 cases of mechanical thrombectomy. From these, data of 620 have been considered well completed and have been entered in this study. Considering the thrombectomy itself, three groups have been differentiated: Uncomplicated cases, those presenting complications, and those in which some incidence has been noted.

Results Out of 620 thrombectomies included in this review, no incidents have been recorded in 499 (80\%) cases. A total of 135 incidences or complications have been noted (some patients had more than one). These were distributed as follows: $25(4 \%)$ cases of haemorrhage or contrast extravasation, $18(2,9 \%)$ cases of arterial dissection, $15(2,4 \%)$ cases of puncture site hematoma, 1 patient $(0,16 \%)$ presented a carotid-cavernous fistula, $5(0,8 \%)$ cases of in-stent thrombosis, significant embolic events were noted in $43(6,9 \%)$ patients, $24(3,9 \%)$ related to the same territory of the thrombus location, and $19(3 \%)$ extending to another territory. Severe vasospasm has been recorded in $7(1,1 \%)$ cases, material related in $8(1,3 \%)$, and general complications such as allergic reaction or heart arrest in $6(1 \%)$ of cases. Mortality directly attributed to the technique has been considered in $11(1,8 \%)$ cases, most of them in keeping with arterial rupture.

Conclusion Technical and angiographical incidences as well as complications are relatively frequent in mechanical thrombectomy. Among other factors, this can be attributed to patient's age, to the fact of needing a blind access to distal vessels, to the embolic material itself or to vascular atheromatous occlusion. Most of these complications, however, are transient or very much associated to the disease, and the fact of overcoming them is linked to the general state of the patient. Mortality related to the thrombectomy itself in our series has been recorded in less than $2 \%$ of cases. 
Disclosures M. de Miquel: None. L. Aja: None. S. Aixut: None. R. Barranco: None. O. Chirife: None. P. Cardona: None. H. Quesada: None. B. Lara: None. A. Paipa: None.

\section{E-128 DISRUPTING CLOT ADHESION TO THE VESSEL WALL IS AN ESSENTIAL STEP OF DIRECT THROMBUS ASPIRATION}

L Miskolczi*. Holy Cross Hospital, Ft. Lauderdale, FL

10.1136/neurintsurg-2019-SNIS.203

Introduction There are some misconceptions and misunderstandings regarding various implementations of what is generally called A Direct Aspiration First Pass Technique (ADAPT). Variants of the technique may result in significantly different results. We propose a modification of the ADAPT technique that promises faster procedures because of higher rates of single-pass recanalization.

Materials and methods Three years (2016-2018) of prospectively collected data on 147 consecutive thrombectomies by a single operator were reviewed retrospectively that included 68 ADAPT cases. The ratio of stent retriever cases versus ADAPT cases were compared. During this 3 -year period a new catheter handling technique has been implemented, that intends to mobilize the clot before retrieval.

Video animations suggest transcatheter aspiration of a Jellolike substance via the tubing into a canister. In real life that rarely happens. Instead, the goal is to have the embolus wedged, corked into the distal tip of the catheter with the help of vacuum, then to physically remove it along with the catheter, preferably in one piece. We recognized that vacuum alone is frequently insufficient to grab, mobilize and remove the embolus in one piece. Some extra effort, or force, is needed to disrupt the adhesion of the clot to the vessel wall it is wedged in. The pulling force is limited by the vacuum lock between the catheter tip and the embolus. However, a pushing force can be stronger, achieving two goals simultaneously: disrupt the clot adhesion, and help stronger corking of the embolus into the catheter tip where the vacuum will be able to hold it stronger during removal.

The guide sheath is advanced as high as possible to provide better support. The aspiration catheter is then advanced right in front of the embolus and vacuum is turned on. The catheter is then pushed forward, beyond the original position of the embolus, during continuous aspiration. If the catheter moves forward with ease, the embolus is almost certainly mobilized and locked. The catheter can be immediately removed.

The success or failure of this forward-push technique was correlated with ADAPT ONLY success versus cross-over to stent retriever, based on our fluoroscopic videos.

Results During the 3-year period the ratio of ADAPT ONLY cases grew from $35 \%$ to $61 \%$. The ratio of ADAPT attempts, eventually crossed over to stent retriever, decreased from $46 \%$ to $18 \%$. Forward-push technique was used in 4,13 and 24 cases in the 3 subsequent years. None of them (0 of 41) required crossing over to stent retriever. During the same 3 years forward-push technique was not used or failed in 26, 20 and 20 cases respectively, of them 17 (65\%), $12(60 \%)$ and $10(50 \%)$ required crossing over to stent retrievers.

Conclusion Adhesion disruption using the forward-push technique helps to mobilize the embolus and to prepare it for a more efficient, successful removal in a single piece. Further testing, including bench-top evaluation will be needed to optimize the technique.

Disclosures L. Miskolczi: None.

\section{E-129 THROMBECTOMY IN DISTAL VESSEL OCCLUSION: MCA M2-3 JUNCTION AND BEYOND}

B Donegan*, J Loeb, C Martin, W Holloway, J Halpin, N Akhtar. Saint Luke's Marion Bloch Neuroscience Institute, Kansas City, MO

10.1136/neurintsurg-2019-SNIS.204

Introduction/Purpose Since 2012, our team of neurointerventional physicians have performed over 600 intracranial arterial thrombectomies with stent-retrievers. Thrombectomy in the ICA and M1 segments has been shown in randomized trials to reduce permanent disability and is now the standard of care. The time window has continued to expand. However, less is known about outcomes for thrombectomy performed in distal branches, such as near the M2-3 junction or within the M3 segments. Current guidelines state that thrombectomy in these locations may be performed in carefully selected patients, however data is limited.

Materials and methods A retrospective review of our stroke database from 2012-2018 was undertaken to find cases of MCA interventions involving the distal third of the M2 insular segment or the M3 opercular segment. Isolated ICA, M1 and proximal M2 branch occlusions, as well as proximal occlusions that resulted in distal emboli post-thrombectomy, were excluded. Patients were selected for intervention based on noncontrast CT head, CTA, CT perfusion results; onset time; and clinical exam. Distal vessel thrombectomies were performed with the use of a stent-retriever device. Pre-intervention scans were compared to post-intervention scans to evaluate degree of infarct. The baseline and discharge National Institute of Health Stroke Scale, and the modified Rankin Scale at 90 days were used to evaluate the degree of residual deficits.

Results A total of 43 patients met the inclusion criteria. Mean age was 69.4 (IQR 60.5-82). 23 (53.5\%) were female. Thrombus was categorized as being located completely within the M3 segment in 21 cases (48.8\%) and spanning the M2M3 junction in 22 cases. 6 patients (14.0\%) presented with tandem vessel occlusions involving the ICA or a proximal MCA segment, or proximal occlusion that had moved distally between CTA and angiography. 18 patients received intravenous tPA $(41.9 \%) .42$ cases were treated with a stent-retriever, and 1 case was treated with intra-arterial tPA. Multiple devices were used, however all were $3-4 \mathrm{~mm}$ in diameter, and 39 were $15-20 \mathrm{~mm}$ in length. 3 cases used a $4 \times 40 \mathrm{~mm}$ device. The mean number of passes with a device was 1.2 (range 13). Near or complete reperfusion (mTICI score $2 \mathrm{~b}$ or greater) was achieved in 40 cases $(93.0 \%)$. Median initial NIHSS on presentation was 10.5 (IQR 7.8-18). Median discharge NIHSS was 2 (IQR 1-5.3). Initial and discharge NIHSS was not available in 3 patients. 90-day mRS was $0-2$ in 17 patients (48.6\% of those available) and $3-6$ in 18 patients $(51.4 \%$ of those available). 90-day mRS was not available in 9 patients (18.6\% of total), mostly in the earliest cases found in the database. 3 cases of intraparenchymal hemorrhage occurred (7.0\%), 2 of which were symptomatic including 1 resultant death. 